\title{
Tumor rejection by the poliovirus receptor family ligands of the DNAM-1 (CD226) receptor
}

Short Title: Memory T cell induction by DNAM-1 ligands

Satoko Tahara-Hanaoka ${ }^{1}$, Kazuko Shibuya ${ }^{1}$, Hirayasu Kai ${ }^{1}$, Akitomo Miyamoto ${ }^{1,3}$, Yoshihiro Morikawa ${ }^{4}$, Nobuhiro Ohkochi ${ }^{2}$, Shin-ichiro Honda ${ }^{1}$ and Akira Shibuya ${ }^{1,5}$

${ }^{1}$ Department of Immunology, Institute of Basic Medical Sciences and Center for TARA, ${ }^{2}$ Department of Surgery, Institute of Clinical Medicine, Graduate School of Comprehensive Human Sciences, University of Tsukuba, Ibaraki 305-8575, Japan.

${ }^{3}$ Subteam for Manipulation of Cell Fate, BioResource Center, RIKEN Tsukuba Institute, Ibaraki 305-0074, Japan

${ }^{4}$ Department of Anatomy and Neurobiology, Wakayama Medical University, Wakayama 641-8509, Japan

This research was supported in part by the grants provided by the Ministry of Education, Science and Culture of Japan (to S.T-H., K.S. and A.S.), Special Coordination Funds of the Science and Technology Agency of the Japanese Government (to A.S.), the Uehara Memorial Foundation (to A.S.) and the Yasuda Memorial Foundation (to A.S.).

${ }^{5}$ Correspondence should be addressed to Akira Shibuya, M.D., Ph.D.

Department of Immunology, Institute of Basic Medical Sciences, Graduate School of Comprehensive Human Sciences, University of Tsukuba, 1-1-1, Ten-nodai, Tsukuba, Ibaraki 305-3585, Japan (e-mail; ashibuya@md.tsukuba.ac.jp, FAX: 81-29-853-3410, Phone: 81-29-853-3474)

Total word counts: 4054

Scientific heading; Immunobiology 


\section{Abstract}

The poliovirus receptor CD155 and its family member CD112 (nectin-2) are the ligands for the activating cell surface receptor DNAM-1 on CD8+ T cells and NK cells. Here, we demonstrated that, while the RMA tumor grew in syngeneic mice, DNAM-1 ligands-transduced RMA was rejected, in which CD8+ T cells and NK cells played an essential role. Importantly, CD8+ memory cytotoxic T cells to parental RMA were generated in these mice. We found that DNAM-1 was also expressed on CD8 $\alpha+$, rather than CD8 $\alpha$-, dendritic cells. Cross-linking DNAM-1 induced maturation of CD8 $\alpha+$ dendritic cells. Antigen presentation by these stimulated dendritic cells drived Th1 cells. Moreover, the rejection of DNAM-1 ligands-transduced RMA was canceled in CD4+ $\mathrm{T}$ cell-depleted and MHC class II deficient mice. Taken together, these results suggest that DNAM-1 ligands stimulate innate immunity by CD $8 \alpha+$ dendritic cells as well as NK cells, which efficiently prime cell-mediated tumor specific immunity. 


\section{Introduction}

Immune surveillance is mediated by both cellular and humoral immunities.

Cytotoxic $\mathrm{T}$ lymphocytes (CTL) and natural killer (NK) cells are major players in cell-mediated immunity against tumors or virus-infected cells. CTL and NK cells often share adhesion or costimulatory molecules, including CD2 and leukocyte function-associated antigen-1 (LFA-1; CD11a/CD18), CD27, 2B4, CD28, inducible costimulator (ICOS), NKG2D and others ${ }^{1,2}$. These cell surface molecules not only mediate intercellular binding between these cytotoxic lymphocytes and target cells, but also participate in signal transduction for cytotoxicity and cytokine secretion ${ }^{1,3}$.

DNAM-1 (CD226) is an adhesion molecule that is a member of immunoglobulin superfamily containing two Ig-like domains of the $\mathrm{V}$-set ${ }^{4-6}$. DNAM-1 is a $\sim 65 \mathrm{kDa}$ glycoprotein expressed on the majority of NK cells, T cells, monocytes and platelets and a subset of $B$ lymphocytes ${ }^{4,7,8}$. The monoclonal antibody (mAb) against DNAM-1 inhibited antigen-specific CTL- and NK cell-mediated cytolysis of some, but not all, tumor targets in vitro, suggesting that DNAM-1 is involved in cytotoxicity against certain tumor cells expressing the DNAM-1 ligand. Recently, we and others have identified 
the poliovirus receptor (PVR) CD155 and its family member CD112 (PVR-related family-2 (PRR-2), also called as nectin-2), as the ligands for human DNAM-1 ${ }^{9,10}$. Ectopic expression of human CD155 and/or CD112 rendered tumor cell lines more susceptible to IL-2-activated T and NK cell-mediated cytotoxicity in vitro. While the human DNAM-1 ligands CD112 and CD155 are broadly distributed on epithelial and endothelial cells in many tissues ${ }^{11,12}$, they are over-expressed on certain human tumors, including colorectal carcinomas ${ }^{13}$, myeloid leukemias ${ }^{14}$, and neuroblastomas ${ }^{15}$, suggesting that the DNAM-1 ligands expression is inducible by "stress" such as transformation and infection. However, it has still been an open question how the interaction of DNAM-1 with its ligands is involved in immune responses in vivo.

We have recently identified the murine homologues of DNAM-1 and its ligands 16. In the present study, we investigated an in vivo role of DNAM-1 and DNAM-1 ligands on tumor immunosurveillance. 


\section{Methods}

\section{Mice, antibodies and cells}

C57BL/6 (B6) mice were purchased from Charles River Japan and were bred and housed under specific pathogen-free conditions. OVA peptide (ISQAVHAAHAEINEAGR; OVA 323 - 339)-specific CD4+ TCR transgenic mice (OT-II) and MHC class II-deficient mice were kindly provided from William R. Heath (WEHI, Parkville, Australia) and Diane Mathis (Harvard, Boston), respectively. For depletion of CD8+ and CD4+ T cells and NK cells, anti-mouse CD8 $\alpha$ (19/178) (a gift from Eiichi Nakayama (Okayama University, Japan)), anti-mouse CD4 (GK1.5) and anti-NK1.1 (PK136) (obtained from ATCC (Manassas, VA) were used. The other mAbs used in this study were purchased from BD Biosciences (San Jose, CA). RMA cells stably expressing mouse CD112 $\alpha$ and CD155 (RMA-CD112 and RMA-CD155, respectively) were established, as described previously ${ }^{16}$. Control RMA cells were also established by transducing $\mathrm{pMX}$ vector alone and this transduction was confirmed by genomic PCR for the vector backbone sequence, using the primers combination of 
5'-ggtggaccatcctctagact-3' and 5'-cccttttctggagactaaat-3'. Anti-mouse DNAM-1 (TX-42) mAb was generated in our laboratory by standard methods ${ }^{16}$. TX42 mAb was a blocking antibody that inhibited binding of DNAM-1-Fc chimeric protein with RMA-CD155 or RMA-CD112, as determined by flow cytometry. In vivo injection (200 $\mu$ g i.p. on day 1 and day 3) of TX42 mAb did not affect the populations of T cells, B cells and NK cells in spleen at day 7, as determined by flow cytometry, while anti-CD8 $\alpha$ (19/178) and anti-NK1.1 (PK136) almost completely depleted CD8+ T cells and NK cells, respectively, by the same procedure. TX42 mAb was free from endotoxin, as examined using Limulus amoebocyte lysate (LAL) test (Sigma).

\section{Tumor growth assay and survival of mice}

Groups of five or ten C57BL/6 mice were pretreated by i.p. injection of $200 \mu \mathrm{g}$ mAb for depletion of CD8+ T, CD4+ T or NK cells at day $-1,1,8,15$ and 22, where day 0 is the day of primary or secondary tumor inoculation. Depletion of each cell type was confirmed by analyzing splenocytes from a group of mice treated with each mAb. For blocking of interaction of DNAM-1 with its ligands in vivo, mice were pretreated by 
i.p. injection of $200 \mu \mathrm{g}$ anti-DNAM-1 at day $-1,1,6$ and 10 . Control mice received the equivalent amounts of normal mouse IgG. At day 0 , groups of five or ten mice per experiments were injected subcutaneously in the back with $1 \times 10^{5}$ RMA cells which were transduced with $C D 112 \delta, C D 155$ or mock-control vector. Mice were examined twice a week for tumor size with a caliper square. For the re-challenge experiments, mice that had completely rejected the initial tumor cells were injected with mock-transduced RMA tumor cells 70 days after the first tumor inoculation.

\section{ELISPOT assay}

ELISPOT assay was performed with a mouse IFN- $\gamma$-specific ELISPOT kit (e-Bioscience, San Diego, CA) according to the manufacturer's instructions. Splenocytes from naïve C57BL/6 mouse were used as an antigen presenting cells (APC) after pulse with $1 \mu \mathrm{M}$ gagL peptide (CCLCLTVFL; gagL ${ }_{75-83}$ ), which is a leader sequence of Rauscher murine leukemia virus, or a control OVA peptides (SIINFEKL; OVA $257-264$ ) for $30 \mathrm{~min}$ at $37^{\circ} \mathrm{C}$, and then plated at $5 \times 10^{6}$ cells/well. CD8+ T cells from splenocytes of mice inoculated with RMA-CD155 or RMA-mock were purified by positive selection using MACS (Miltenyi Biotech, Auburn, CA) according to the manufacturer's instructions, and plated across a range of cell concentrations to achieve 10 to 100 spots/well for accurate counting, and cultured for 24 hours with APC 
pre-pulsed with peptides. The colored spots were counted using a stereomicroscope. Frequencies of antigen-specific IFN- $\gamma$-secreting cells were calculated based on the numbers of CD8+ cells and the number of spots per well after subtraction of background, which was obtained by incubating CD8+ T cells stimulated with the control OVA peptides. Experiments were performed in triplicate.

\section{In vitro stimulation of DC}

CD11c+ DC was purified from spleen cells of C57BL/6 mice by positive selection using MACS (Miltenyi Biotech, Auburn, CA). Cells were incubated with anti-CD16/32 mAb (2.4G2) to block non-specific binding to Fc $\gamma$ receptors by antibodies and then stimulated with plate-coated control Ig or anti-mDNAM-1 (TX-42) for 4 days. Cells were then stained with PE-conjugated anti-CD11c mAb, APC-conjugated anti-CD8 mAb and either FITC-conjugated anti-CD80, anti-CD86, anti-MHC class II (I-Ab) mAbs or control IgG and analyzed by flow cytometry. For analysis of function of DNAM-1-expressing DC, CD11C+CD8 $\alpha+$ or $C D 11 c+C D 8 \alpha-$ DCs were purified from splenocytes of C57BL/6 mice by positive selection by using MACS and then by flow cytometry. Each DC population was stimulated with plate-coated anti-DNAM-1, anti-CD40 or control Ig for 4 days. Cells were pulsed with the OVA peptide and then 
co-cultured with CD4+ T cells from splenocytes of OT-II mice for 7 days. Cells were then stimulated with anti-CD3 and anti-CD28 for 48 hours, and IFN- $\gamma$ concentrations in the culture supernatants were determined with a mouse IFN- $\gamma$-specific ELISA kit (Biosource International, Camarillo, CA).

\section{Immunohisotochemistry}

Immunostaining was performed with some modifications as previously described ${ }^{17}$. Six-micrometer cryostat sections were processed for immunohistochemical procedures based on the Labeled Polymer method (DAKO Japan, Kyoto, Japan). Briefly, sections were treated with $3 \%$ hydrogen peroxidase in absolute methanol for 30 minutes to quench endogenous peroxidase activity. After antigen retrieval with Target Retrieval Solution (DAKO Japan), sections were preincubated in $0.01 \mathrm{M}$ PBS containing 5\% normal goat serum (Jackson Immuno Research, West Grove, PA) at room temperature (RT) for $1 \mathrm{hr}$, and then incubated in anti-PRR-2 mAb (1:20 dilution) containing $0.5 \%$ bovine serum albumin (BSA) at RT for 2 hrs. After washes with $0.1 \mathrm{M}$ PBS, the sections were incubated in goat anti-mouse 
Ig conjugated to peroxidase-labeled dextran polymer (EnVision+, DAKO Japan) at RT for 30 min. After washes with 0.1M PBS, the horseradish peroxidase reaction was developed in $0.1 \mathrm{M}$ Tris-buffered saline, $\mathrm{pH} \quad 7.4$, containing $0.05 \%$ 3,3'-diaminobenzidine tetrahydrochloride (Sigma), $0.02 \%$ nickel sulfate, and $0.01 \%$ hydrogen peroxide. Methyl green was used for counterstaining. The following controls were performed: (i) incubation with isotype control antibody instead of the primary antibody; (ii) incubation without the primary antibody or without primary and secondary antibodies. All controls revealed no signals.

\section{Quantitative RT-PCR}

Total RNA was extracted from gastric cancer tissues and the adjacent normal tissues in three patients with poorly-differentiated gastric cancer with ISOGEN reagent (Nippongene, Japan). Reverse transcription was performed with $5 \mu \mathrm{g}$ of total RNA using Thermoscript RT-PCR System and $50 \mathrm{ng}$ of random hexamers (Invitrogen) in a final volume of $20 \mu \mathrm{l}$. Real-time PCR analysis of CD112 was carried out on an ABI7700 sequence detector (Applied Biosystems, Foster City, CA). The primers for 
CD112 were as follows: upper primer, 5'- atg tgg ccg cct tcc acc cta a-3'; lower primer, 5'-cag act gtc cac tgc gtg gat $g$-3'. The $18 \mathrm{~S}$ rRNA level was measured as an internal control (upper primer, 5'-ccc tgc cct ttg tac aca cc-3'; lower primer, 5'-cga tcc gag ggc ctc act a-3'). The thermal cycling conditions comprised an initial denaturation step at $95^{\circ} \mathrm{C}$ for $10 \mathrm{~min}$, followed by 40 cycles at $95^{\circ} \mathrm{C}$ for $15 \mathrm{~s}$ and $60^{\circ} \mathrm{C}$ for $1 \mathrm{~min}$. Standard curves were generated using serial dilutions of cDNA from HOS cells (human osteosarcoma cell line), covering 4 orders of magnitude and yielding correlation coefficients of at least 0.98 in all experiments. Each standard and sample value was determined in triplicate in three independent experiments. In each experiment, CD112 expression under a given experimental condition was calculated relative to the control condition. 


\section{RESULTS}

\section{Expression of the DNAM-1 ligand on human poorly-differentiated gastric cancer}

Recent evidences indicate that the DNAM-1 ligands CD155 and CD112 are over-expressed on certain human tumors, including colorectal carcinomas, neuroblastomas and myeloid leukemias ${ }^{13-15}$. We also investigated the expression of the DNAM-1 ligand CD112 on specimens from five patients with gastric cancer, including two well-differentiated and three poorly-differentiated histological types, and two patients with colon cancer. Immunohistochemical analyses demonstrated that CD112 was over-expressed preferentially in the poorly-, but not well-, differentiated histological type of gastric cancer (Fig 1a). We could not clearly detect CD112 in the untransformed normal tissue area bordering transformed cancer tissue in each specimen. To further assess whether CD112 is specifically expressed in poorly-differentiated cancer tissue but not in normal gastric tissue, we divided the gastric tissues resected under surgery into transformed and normal areas of slices and compared mRNA expression of CD112 in each patient by quantitative RT-PCR analysis. As shown in Fig $\mathbf{1 b}$, relative amount of CD112 mRNA expressions are about three 
times higher in the poorly-differentiated gastric cancer compared with that in normal gastric tissue in all the three patients. We also observed the over-expression of CD112 on colon cancer as well (Fig 1a). These observations, along with the recent reports ${ }^{13-15}$, suggest that DNAM-1 ligands can be targets for tumor immunosurveillance in vivo.

\section{RMA tumor expressing DNAM-1 ligands is rejected in mice}

To investigate the in vivo consequences of DNAM-1 ligands expression on tumor cells, B6 mice were inoculated subcutaneously with a murine T cell lymphoma RMA transfected with control vector (RMA-mock) or CD112 (RMA-CD112). Whereas mice injected with $1 \times 10^{5}$ RMA-mock all developed tumors and died by 55 days after tumor inoculation, most mice rejected the same number of RMA-CD112 (Fig 2A). These recipient mice of RMA-CD112 survived significantly longer than those of RMA-mock (Fig 2A). Similarly, most mice rejected $1 \times 10^{5}$ RMA trasfected with CD155 (RMA-CD115) and survived more than 175 days (Fig 2B). Since all these RMA transfectants showed similar growth kinetics in vitro, as determined by BrdU 
incorporated proliferation assay (data not shown), these results indicate that ectopic expression of DNAM-1 ligands can result in rejection of tumors in syngeneic mice.

CD8+ $T$ cells are responsible for the rejection of DNAM-1 ligands-transduced RMA cells

DNAM-1 is strongly expressed on the majority of CD8+ T cells and mediates a costimulatory signal in antigen-specific CD8+ $\mathrm{T}$ cells in vitro ${ }^{4,16}$. We therefore investigated whether CD8+ $\mathrm{T}$ cells were responsible for the rejection of RMA expressing DNAM-1 ligands. Mice were treated in vivo with depleting monoclonal antibodies specific for CD8 or with a control antibody and then inoculated with RMA transfectants. Depletion of CD8+ T cells completely canceled the tumor rejection of both RMA-CD155 and RMA-CD112, and these mice became moribund at the same time as recipients of RMA-mock (Fig 2C, D). These results indicated that CD8+ T cells were essential for the rejection of DNAM-1 ligands-transduced RMA tumors.

Colonna and colleagues have recently identified the T cell-activated increased late expression (Tactile) (also called as CD96), which is expressed on activated T cells 
${ }^{18}$, as another receptor ligand for human CD155 ${ }^{19}$. However, it is unclear whether the mouse homologue of CD96 is also a receptor for mouse CD155 or CD112, although it has not yet been identified. To confirm that DNAM-1, but not putative murine CD96, is responsible for the rejection of DNAM-1-ligands-expressing RMA tumors, we generated an anti-DNAM-1 mAb (TX42) that was able to block the ligand binding in vitro (Fig 3A). Mice were pretreated in vivo with TX42 mAb, which did not deplete T cells and NK cells in vivo (Fig 3B), and then inoculated subcutaneously with RMA-CD155. Eight days after tumor inoculation when tumors had not yet grown enough to be visible on the skin, the skin regions was resected and subjected to histological analyses. Whereas RMA-mock substantially proliferated in the skin region, most RMA-CD155 appeared to be dead (Fig 3C). However, pretreatment with anti-DNAM-1 mAb restored RMA-CD155 growth (Fig 3C), and the survival of these mice was significantly shorter than that of mice pretreated with control Ig (Fig 3D). Similar results were observed in mice inoculated with RMA-CD112 (data not shown). Because TX42 mAb did not affect the lymphocytes population in vivo, these results suggested an essential role of DNAM-1 on CD8+ T cells in the tumor rejection. 
Induction of memory CD8+ $T$ cells in mice inoculated with DNAM-1 ligand-expressing RMA

To investigate whether DNAM-1 ligands expression on RMA induces T cell-mediated immunological memory, mice that had previously rejected the CD155-tranfected RMA tumor cells were re-challenged with the parental RMA tumors 70 days after the first inoculation (Fig 4). Whereas RMA-mock grew and killed all naïve mice after the first inoculation, they were rejected in all the mice that had once rejected RMA-CD155 (Fig 4). However, depletion of CD8+ T cells with in vivo injection of anti-CD8 mAb before the re-challenge canceled the rejection of RMA (Fig 4). These results suggest that CD8+ memory $T$ cells specific for an RMA tumor-associated antigen were generated in mice inoculated with RMA-CD155.

RMA is a Rauscher murine leukemia virus-transformed cell line bearing $H-2 D^{b}$,

which associates with a peptide derived from the gag leader (gagL) sequence of the virus recognized by CTL as an epitope ${ }^{20,21}$. To investigate whether CD8+ T cells generated in mice inoculated with RMA-CD155 were specific to the gagL peptide, we 
isolated CD8+ $T$ cells from spleen in mice after inoculation with RMA-mock or RMA-CD155, stimulated the CD8+ $\mathrm{T}$ cells in vitro with the gagL peptide and determined the frequency of the peptide-specific IFN- $\gamma$ producing CD8+ T cells by ELISPOT assay. Although we could not detect CD8+ $\mathrm{T}$ cells that responded to the gagL peptide in mice immunized with RMA-mock or RMA-CD155 at the priming phase around day 3 5 after the tumor challenge by this assay (data not shown), mice inoculated with RMA-CD155 generated CD8+ T cells responsive to the gagL-peptide significantly more than those inoculated with RMA-mock at day 14 to 18 when RMA-mock tumor become visible (Fig 4B). These results suggested that the gagL peptide-specific CD8+ T cells might play an important role for efficient tumor rejection of RMA-CD155 and memory CTL induction.

\section{NK cells are also involved in the tumor rejection}

DNAM-1 is expressed on NK cells and involved in NK cell-mediated cytotoxicity and cytokine secretion in vitro ${ }^{4,9}$. We therefore examined whether NK cells are also involved in the rejection of RMA-CD155 and RMA-CD112 that express 
MHC class I $\left(H-2^{b}\right)$. As demonstrated in Figs 2E and $\mathbf{F}$, depletion of NK cells with anti-NK1.1 mAb partially canceled the tumor rejection of RMA-CD112 and RMA-CD155, indicating that NK cells were in part involved in the tumor rejection. These results suggested that NK cells might be directly activated by DNAM-1 ligand-expressing RMA.

\section{DNAM-1-mediated signal induces maturation of CD8 $\alpha+$ dendritic cells}

In a detailed study of mouse DNAM-1 expression on immune cells using our newly generated anti-DNAM-1 mAb, we found that a subset of CD11c+ cells also expressed DNAM-1. Further examination demonstrated that DNAM-1 was preferentially expressed on CD8 $\alpha+$, rather than CD8 $\alpha-$, CD11c+ dendritic cells (DC) in spleen (Fig 5A), suggesting that DNAM-1 ligands may stimulate also this subset of DC. To examine this possibility, CD11c+ cells were purified from spleen by MACS sorting, stimulated with plate-coated anti-DNAM-1 mAb and then stained with anti-CD11c, anti-CD8 $\alpha$ and either anti-CD80, anti-CD86 or anti-MHC class II. As demonstrated in Fig 5B, cross-linking DNAM-1 with anti-DNAM-1 mAb induced up-regulation of CD80, 
CD86 and MHC class II expressions in CD8 $\alpha+$, but not CD8 $\alpha-$, CD11c+ cells, indicating that DNAM-1-mediated signal induces maturation of CD8 $\alpha+$ DC.

\section{CD4+ T cells are also involved in the tumor rejection}

CD8 $\alpha+$ DC is a functionally specialized subpopulation (reviewed in Shortman, $\mathrm{K}$., et $\mathrm{al}^{22}$ ), which produces much higher level of IL-12 p70 than CD8 $\alpha$ - DC population and is involved in Th1 differentiation from naïve helper $\mathrm{T}$ cells ${ }^{23,24}$. Therefore, we assumed that antigen presentation by MHC class II of these stimulated CD8 $\alpha+D C$ drives differentiation of antigen-specific helper $\mathrm{T}$ cells into Th1 cells. To test this hypothesis, CD4+ $T$ cells purified from OVA-specific TCR transgenic mice were co-cultured with OVA-peptide-pre-pulsed CD8 $\alpha+$ or CD8 $\alpha-$ DC that had been stimulated with anti-DNAM-1 mAb. We observed that interferon (IFN)- $\gamma$ secretion was significantly increased in culture of CD4+ T cells with CD8 $\alpha+$, but not CD8 $\alpha-$, DC stimulated with anti-DNAM-1 mAb (Fig 5C), indicating that DNAM-1 on CD8 $\alpha+$ DC is involved in Th1 polarization. These results suggested that not only CD8+ T cells but also CD4+ T cells might play an important role for tumor rejection of RMA-CD112 or 
RMA-CD155. In fact, depletion of CD4+ T cells with anti-CD4 mAb partially canceled the rejection of RMA-CD112, leading to shorter survival of mice without CD4+ T cells than those with CD4+ T cells (Fig 2A, G). Similarly, the survival of mice deficient in MHC class II was significantly shorter than those of wild type mice after inoculation with RMA-CD112 (Fig 2A, F). 


\section{DISCUSSION}

The DNAM-1 ligands are constitutively expressed at low levels on epithelial and endothelial cells. In the present study, we have shown that the DNAM-1 ligand CD112 is over-expressed on human gastric and colon cancers. There are also evidences that the DNAM-1 ligands expression is up-regulated on human tumors, including colorectal carcinomas, neuroblastomas and myeloid leukemias ${ }^{13-15}$. These observations suggest that the DNAM-1 ligands expression may be inducible by "stress" such as transformation, leading us to examine whether these 'stress-inducible self-ligands' may potentially stimulate innate and/or adaptive tumor immunity. Here, we have demonstrated that ectopic expression of DNAM-1 ligands permitted rejection of RMA tumors inoculated in mice. CD8+ $T$ cells played an essential role for the rejection of the DNAM-1 ligand-expressing RMA tumors.

Several lines of evidences have demonstrated that innate immune surveillance is important for efficient priming of adaptive anti-tumor immunity ${ }^{25}$. Several cell surface receptors, including CD27 and NKG2D, expressed on NK cells have been reported to induce the primary and secondary tumor rejections by NK cells 
and CD8+ memory $\mathrm{T}$ cells, respectively ${ }^{26,27}$, suggesting a link between innate and adaptive immunities. Although both human and mouse DNAM-1 are expressed on NK cells and involved in NK cell-mediated cytotoxicity and interferon (IFN)- $\gamma$ secretion in vitro ${ }^{4,9}$, it remained undetermined whether DNAM-1ligands on tumors that bear MHC class I could stimulate NK cells in vivo in spite of inhibitory signals in NK cells mediated by MHC class I receptors. In the present study, we have demonstrated that, although RMA tumor expresses $\mathrm{MHC}$ class I $\left(\mathrm{H}-2^{\mathrm{b}}\right)$, NK cells seemed to be partially responsible for rejection of RMA expressing the DNAM-1 ligands. It was likely that NK cells were directly activated by DNAM-1 ligand-expressing RMA tumors and produced cytokines, such as interferon (IFN)- $\gamma$ and/or IFN- $\alpha$ a . an efficient priming of adaptive immunity.

One of the most notable findings in the present study was that $\operatorname{CD} 8 \alpha+$, but not CD8 $\alpha-$, DC substantially expressed DNAM-1 and cross-linking DNAM-1 with anti-DNAM-1 mAb induced maturation of CD8 $\alpha+$ DC in vitro. Importantly, the CD8 $\alpha+$ DC is a functionally specialized subpopulation (reviewed in ${ }^{22}$ ). They produces much higher level of IL-12 p70 than CD8 $\alpha$ - DC populations ${ }^{23,24}$, resulting in differentiation of 
Th1 cells that facilitate cell-mediated immunity. In fact, our study showed that antigen presentation by $C D 8 \alpha+$ DC stimulated with anti-DNAM-1 drived Th1 cells. It was of note, however, that depletion of CD4+ T cells or deficiency of MHC class II did not completely canceled the tumor rejection of RMA-CD112, indicating that CD4+ T cells are not necessarily required for the tumor rejection. Recent evidences have demonstrated that $\mathrm{CD} 8 \alpha+\mathrm{DC}$ selectively endocytosis dying cells in vivo ${ }^{28}$, and can cross-present exogenous antigens on MH C class I ${ }^{29,30}$, resulting in priming $\mathrm{CTL}^{31}$. Therefore, it was also likely that the CD8 $\alpha+$ DC carried out the uptake of tumor debris destroyed by NK cells and directly stimulated CD8+ $T$ cells resulted by cross-presentation of a tumor-associated antigen.

We have demonstrated that CD8+ $T$ cells specific to the Rauscher murine leukemia virus-derived gag $L$ peptide antigen were generated in mice immunized with DNAM-1 ligand-transduced RMA significantly more than those immunized with mock-transduced RMA. Moreover, cross-linking DNAM-1 induced maturation of CD8 $\alpha+$ dendritic cells. Antigen presentation by these stimulated dendritic cells drived Th1 cells and CD4+ helper T cells were involved in tumor rejection. Taken together, 
these results suggested that DNAM-1 ligand expression on RMA may play an important role for priming of the gagL peptide-specific CD8+ CTL. However, we still cannot reach this conclusion by these data at present, because we could not directly detect CD8+ T cells that responded to the peptide in the early days of priming around day 3 5 after tumor challenge by the ELISPOT assay, and therefore could not exclude a possibility that proliferation of the gagL peptide-specific CD8+ T cells were affected in mice inoculated with RMA-mock by an immuno-suppressive effect of growing RMA tumor. Nonetheless, the present studies suggested that interaction of DNAM-1 and DNAM-1 ligands played an important role for tumor rejection mediated by the RMA tumor-associated antigen-specific CD8+ T cells. Although we observed most tumor cell lines, except RMA, expressed the DNAM-1 ligands, whether these tumors are rejected in vivo may depend on expression of tumor antigen specifically recognized by CD8+ T cells.

In summary, the present study proposes a model that DNAM-1 ligands, whose expression are inducible on tumors, potentially trigger innate immunity mediated by NK cells and CD8 $\alpha+\mathrm{DC}$, both of which further stimulate each other by cytokines produced 
by themselves such as IFN- $\alpha$, IFN- $\gamma, \mathrm{IL}-12, \mathrm{IL}-15$ and IL-18 ${ }^{32}$. CD $8 \alpha+\mathrm{DC}$ then captures tumor debris destroyed by NK cells and present a tumor-associated antigen on both MHC class I and class II, resulting in priming antigen-specific CD8+ and CD4+ $\mathrm{T}$ cells. Further studies should be required to clarify molecular and cellular mechanisms of DNAM-1-mediated tumor immunity.

\section{Acknowledgement}

We thank L. Lanier and Mitsuyasu Kato for helpful discussions. We also thank Ayumi Hara, Yuko Onoda and Hua Zhang for technical assistances and Yurika Soeda for secretarial assistance. 


\section{References}

1. Springer TA. Adhesion receptors of the immune system. Nature. 1990;346:425-434.

2. Lanier LL. Turning on natural killer cells. J Exp Med. 2000;191:1259-1262.

3. Dustin ML, Springer TA. Role of lymphocyte adhesion receptors in transient interactions and cell locomotion. Annu Rev Immunol. 1991;9:27-66.

4. Shibuya A, Campbell D, Hannum C, et al. DNAM-1, a novel adhesion molecule involved in the cytolytic function of T lymphocytes. Immunity. 1996;4:573-581.

5. Shibuya A, Lanier LL, Phillips JH. Protein kinase C is involved in the regulation of both signaling and adhesion mediated by DNAX accessory molecule-1 receptor. J Immunol. 1998;161:1671-1676.

6. Shibuya A, Tahara-Hanaoka S, Shibuya K. DNAM-1 (CD226), a two-sword fencer for innate and adaptive immunity. Curr Med Chem-Anti-Inflamatory Allergy Agents. 2005;4:53-58.

7. Burns GF, Triglia T, Werkmeister JA, Begley CG, Boyd AW. TLiSA1, a human T lineage-specific activation antigen involved in the differentiation of cytotoxic T lymphocytes and anomalous killer cells from their precursors. J Exp Med. 1985;161:1063-1078.

8. Kojima H, Kanada H, Shimizu S, et al. CD226 mediates platelet and megakaryocytic cell adhesion to vascular endothelial cells. J Biol Chem. 2003;278:36748-36753.

9. Tahara-Hanaoka S, Shibuya K, Onoda Y, et al. Functional characterization of DNAM-1 (CD226) interaction with its ligands PVR (CD155) and nectin-2 (PRR-2/CD112). Int Immunol. 2004;16:533-538.

10. Bottino C, Castriconi R, Pende D, et al. Identification of PVR (CD155) and Nectin-2 (CD112) as cell surface ligands for the human DNAM-1 (CD226) activating molecule. J Exp Med. 2003;198:557-567.

11. Lopez M, Aoubala M, Jordier F, Isnardon D, Gomez S, Dubreuil P. The human poliovirus receptor related 2 protein is a new hematopoietic/endothelial homophilic adhesion molecule. Blood. 1998;92:4602-4611.

12. Iwasaki A, Welker R, Mueller S, Linehan M, Nomoto A, Wimmer E. Immunofluorescence analysis of poliovirus receptor expression in Peyer's patches of humans, primates, and CD155 transgenic mice: implications for poliovirus infection. J Infect Dis. 2002;186:585-592.

13. Masson D, Jarry A, Baury B, et al. Overexpression of the CD155 gene in human 
colorectal carcinoma. Gut. 2001;49:236-240.

14. Pende D, Spaggiari GM, Marcenaro S, et al. Analysis of the receptor-ligand interactions in the natural killer-mediated lysis of freshly isolated myeloid or lymphoblastic leukemias: evidence for the involvement of the Poliovirus receptor (CD155) and Nectin-2 (CD112). Blood. 2005;105:2066-2073.

15. Castriconi R, Dondero A, Corrias MV, et al. Natural killer cell-mediated killing of freshly isolated neuroblastoma cells: critical role of DNAX accessory molecule-1-poliovirus receptor interaction. Cancer Res. 2004;64:9180-9184.

16. Tahara-Hanaoka S, Miyamoto A, Hara A, Honda S, Shibuya K, Shibuya A. Identification and characterization of murine DNAM-1 (CD226) and its poliovirus receptor family ligands. Biochem Biophys Res Commun. 2005;329:996-1000.

17. Rump A, Morikawa Y, Tanaka M, et al. Binding of ovarian cancer antigen CA125/MUC16 to mesothelin mediates cell adhesion. J Biol Chem. 2004;279:9190-9198. 18. Wang PL, O'Farrell S, Clayberger C, Krensky AM. Identification and molecular cloning of tactile. A novel human T cell activation antigen that is a member of the Ig gene superfamily. J Immunol. 1992;148:2600-2608.

19. Fuchs A, Cella M, Giurisato E, Shaw AS, Colonna M. Cutting edge: CD96 (tactile) promotes NK cell-target cell adhesion by interacting with the poliovirus receptor (CD155). J Immunol. 2004;172:3994-3998.

20. Sijts AJ, De Bruijn ML, Ressing ME, et al. Identification of an H-2 Kb-presented Moloney murine leukemia virus cytotoxic T-lymphocyte epitope that displays enhanced recognition in H-2 Db mutant bm13 mice. J Virol. 1994;68:6038-6046.

21. Chen W, Qin H, Chesebro B, Cheever MA. Identification of a gag-encoded cytotoxic T-lymphocyte epitope from FBL-3 leukemia shared by Friend, Moloney, and Rauscher murine leukemia virus-induced tumors. J Virol. 1996;70:7773-7782.

22. Shortman K, Liu YJ. Mouse and human dendritic cell subtypes. Nat Rev Immunol. 2002;2:151-161.

23. Maldonado-Lopez R, De Smedt T, Michel P, et al. CD8alpha+ and CD8alphasubclasses of dendritic cells direct the development of distinct T helper cells in vivo. J Exp Med. 1999;189:587-592.

24. Pulendran B, Smith JL, Caspary G, et al. Distinct dendritic cell subsets differentially regulate the class of immune response in vivo. Proc Natl Acad Sci U S A. 1999;96:1036-1041. 25. Smyth MJ, Godfrey DI, Trapani JA. A fresh look at tumor immunosurveillance and 
immunotherapy. Nat Immunol. 2001;2:293-299.

26. Kelly JM, Darcy PK, Markby JL, et al. Induction of tumor-specific T cell memory by NK cell-mediated tumor rejection. Nat Immunol. 2002;3:83-90.

27. Diefenbach A, Jensen ER, Jamieson AM, Raulet DH. Rae1 and H60 ligands of the NKG2D receptor stimulate tumour immunity. Nature. 2001;413:165-171.

28. Iyoda T, Shimoyama S, Liu K, et al. The CD8+ dendritic cell subset selectively endocytoses dying cells in culture and in vivo. J Exp Med. 2002;195:1289-1302.

29. den Haan JM, Lehar SM, Bevan MJ. CD8(+) but not CD8(-) dendritic cells cross-prime cytotoxic T cells in vivo. J Exp Med. 2000;192:1685-1696.

30. Pooley JL, Heath WR, Shortman K. Cutting edge: intravenous soluble antigen is presented to CD4 T cells by CD8- dendritic cells, but cross-presented to CD8 T cells by CD8+ dendritic cells. J Immunol. 2001;166:5327-5330.

31. Belz GT, Smith CM, Eichner D, et al. Cutting edge: conventional CD8 alpha+ dendritic cells are generally involved in priming CTL immunity to viruses. J Immunol. 2004;172:1996-2000.

32. Degli-Esposti MA, Smyth MJ. Close encounters of different kinds: dendritic cells and NK cells take centre stage. Nat Rev Immunol. 2005;5:112-124. 


\section{Figure Legends}

Figure 1. Upregulation of CD112 expression in gastric and colon cancers

(A) Specimens from human gastric and colon cancer tissues were stained with anti-CD112 mAb (TX31). Positive signals were observed in the poorly-differentiated (black arrow), but not well-differentiated (white arrow), transformed epithelial cells in gastric cancer (left). The transformed epithelial cells also show the highly positive signal (black arrow) in colon cancer (right).

(B) mRNA was obtained from gastric cancer tissues from three patients with poorly-differentiated histological type. In each patient, mRNA was also obtained from untransformed normal tissue area. RT-PCR was performed with CD112-specific primers and the amount of cDNA was measured by quantitative PCR analysis. CD112 mRNA expression was normalized to the internal standard 18S ribosomal RNA.

Figure 2. Rejection of DNAM-1 ligands-expressing RMA tumor cells

Groups of five C57BL/6 mice were pretreated with control Ig or monoclonal antibodies 
indicated at day $-1,1,8,15$ and 22 . The mice were inoculated s.c. in the back at day 0 with $1 \times 10^{5}$ mock-transduced RMA tumor cells (open symbols) or CD112- or CD155-transduced RMA tumor cells (closed symbols). Survival data for five mice per group are shown.

Figure 3. DNAM-1 is involved in rejection of DNAM-1 ligands-expressing RMA

\section{tumor cells}

CD155-RMA and CD112-RMA were stained with DNAM-1-Fc chimeric protein, which had been incubated with an excess of TX42 mAb (bold line) or control IgG (thin line), and then analyzed by flow cytometry. Staining with control human IgG1 (dotted line) and blocking of the DNAM-1 Fc staining with an excess of TX42 mAb (bold line) or control IgG (thin line) was performed to show the inhibitory function of TX42 mAb for DNAM-1 ligands recognition (A). Splenocytes from mice were stained with FITC-conjufated anti-CD3 and PE-conjugated DX5 and analyzed 7 days after injection with TX42 mAb (200 $\mu$ g i.p. on day 1 and day 3) by flow cytometry (B). Groups of five C57BL/6 mice were pretreated with control Ig or anti-mDNAM-1 mAb at day -1 . The mice were inoculated s.c. in the back at day 0 with $1 \times 10^{5}$ mock-transduced RMA tumor cells or CD155-transduced RMA tumor cells. The skin regions were subjected to histological analysis with hematoxilin-eosin under light microscopy at day 8. Data 
are representative from three mice in each group (C). Survival data for five mice per group are shown (D).

Figure 4. Memory CD8+ T cell induction specific to RMA by the DNAM-1 ligands

(A) Groups of ten C57BL/6 mice were primarily inoculated with mock-transduced or CD155-transduced RMA tumor cells. Mice that had rejected $1 \times 10^{5}$ CD155-transduced RMA tumor cells were divided into two groups and pretreated (i.p.) either with anti-CD8 $\mathrm{mAb}$ or control Ig, and re-challenged s.c. in the back with $1 \times 10^{5}$ parental RMA tumor cells at 70 days after the primary tumor inoculation. Tumor size in each mouse was measured twice a week and the mean values were plotted.

(B) CD8+ T cells were separated from spleen in mice 14 to 18 days after inoculation with RMA-mock or RMA-CD155 and stimulated in vitro with gagL peptide in the presence of splenocytes that were used as APC. The frequency of the peptide-specific CD8+ T cells, defined as Interferon- $\gamma-$ w determined by ELISPOT assay, as described in Materials and Methods. The error bars indicate 1SD. 
Figure 5. DNAM-1-mediated signal induces maturation and activation of

\section{CD8 $\underline{\alpha D C}$}

(A) Spleen cells from C57BL/6 mice were stained with PE-conjugated anti-CD11c, FITC-conjugated anti-CD8 and biotin-conjugated anti-DNAM-1, followed by APC-conjugated streptavidin. DNAM-1 expressions on CD8 $\alpha-C D 11 c+$ and CD8 $\alpha+C D 11 c+$ cells were analyzed by flow cytometry.

(B) CD11c+ DCs were separated from splenocytes of C57BL/6 mice by positive selection by using MACS. Purified DCs were stimulated for 4 days with plate-coated anti-DNAM-1 mAb or control IgG, and stained with anti-CD11c, anti-CD8 and either control Ig, anti-CD80, anti-CD86 or anti-MHC class II (I-Ab). Mean fluorescence intensity of CD80, CD86 and I-Ab expressions on CD8+CD11c+ or CD8- CD11c+ DCs were determined by flow cytometry. Data are representative in several independent experiments.

(C) $\mathrm{CD} 11 \mathrm{c}+\mathrm{CD} 8 \alpha+$ or $\mathrm{CD} 11 \mathrm{c}+\mathrm{CD} 8 \alpha-\mathrm{DCs}$ were stimulated with plate-coated mAbs indicated for 4 days, pulsed with the OVA peptide and then co-cultured with CD4+ T 
cells from splenocytes of OT-II mice for 7 days. CD4+ T cells were stimulated with anti-CD3 and ant-CD28 for 48 hours and IFN- $\gamma$ concentrations in the culture supernatant were determined by ELISA. Data are representative in several independent experiments. 